\title{
Robot-Assisted Reach Training for Improving Upper Extremity Function of Chronic Stroke
}

\author{
Ki Hun Cho ${ }^{1}$ and Won-Kyung Song ${ }^{1}$ \\ ${ }^{1}$ Department of Rehabilitative and Assistive Technology, Korea National Rehabilitation Research Institute, Korea \\ National Rehabilitation Center, Seoul, Republic of Korea
}

\begin{abstract}
Stroke, as a major risk factor for chronic impairment of upper limb function, can severely restrict the activities of daily living. Recently, robotic devices have been used to enhance the functional upper extremity movement of stroke patients. The purpose of the current study was to assess whether a robotassisted reach training program using a whole arm manipulator (WAM) could improve upper extremity kinematic performance and functional movement for chronic stroke patients. Using a single-group design, this study followed 10 people with chronic stroke (6 men, 61.5 years; Mini-Mental State Examination score: 27.0; onset duration: 8.9 years). WAM with seven degrees of freedom for the shoulder, elbow, and wrist joints was used during robot-assisted reach exercises. Subjects participated in the training program for 40 minutes per day, 2 times a week, for 4 weeks. The main outcome measures were upper extremity kinematic performance (movement velocity) for three directions and functional movement (Action Research Arm Test). Upper extremity kinematic performance and functional movement measures were performed three times: at baseline, during intervention (at 2 weeks), and post intervention. Upper extremity kinematic performance and functional movement showed improvement after two weeks $(P<0.05)$ and four weeks $(P$ $<0.05$ ) of training compared to baseline. The findings of the current study demonstrated the positive effects of short-term robot-assisted reach training on upper extremity kinematic performance as well as functional movement in individuals with chronic stroke. In addition, the findings of the current study may provide valuable information for subsequent randomized controlled trials.
\end{abstract}

Keywords: feedback; rehabilitation; robotics; stroke; upper extremity

Tohoku J. Exp. Med., 2015 October, 237 (2), 149-155. C 2015 Tohoku University Medical Press

\section{Introduction}

Stroke can cause permanent neurological damage or death. Despite the availability of many acute management and treatment techniques, stroke patients live with various functional disabilities that affect daily living activities (Kolominsky-Rabas et al. 2001). Although stroke patients can regain independent walking ability through advanced therapeutic interventions, complete recovery of upper extremity function is uncommon (Nakayama et al. 1994). According to a previous study on the probability of regaining dexterity in flaccid upper extremity, only $11.6 \%$ of stroke patients made a complete functional recovery six months after stroke (Kwakkel et al. 2003). Since most daily living activities are associated with fine motor performance of the upper extremity, recovery of upper extremity function is the primary goal of stroke rehabilitation (Nakayama et al. 1994; Staubli et al. 2009).

In the field of stroke rehabilitation, many potential therapeutic approaches and interventions such as biofeed- back, repetitive task training, constraint-induced movement therapy (CIMT), mirror therapy, brain stimulation, and robot-assisted therapy have been used to restore upper extremity function (Pollock et al. 2014). Among these therapeutic approaches, CIMT and robot-assisted therapy have been demonstrated to be more effective than conventional stroke rehabilitation care (Turner et al. 2013). In recent years, robot-assisted training in particular has been used as a therapeutic intervention to improve upper extremity function of stroke patients (Peter et al. 2011). Robotic devices used for enhancement of upper extremity function are divided into four types of modalities (active, active-assisted, passive, and resistive) according to how force is passed to the paretic arm (Basteris et al. 2014). With these features, robotic devices allow users to participate in interactive training and motor relearning via high-intensity, repetitive, and frequent tasks (Peter et al. 2011; Basteris et al. 2014).

Frisoli et al. (2012) reported that robotic-assisted training with a focus on point-to-point reaching movements was effective in rehabilitating the functional movement of the

Received July 14, 2015; revised and accepted September 7, 2015. Published online October 9, 2015; doi: 10.1620/tjem.237.149.

Correspondence: Won-Kyung Song, Ph.D., Department of Rehabilitative and Assistive Technology, Korea National Rehabilitation

Research Institute, Korea National Rehabilitation Center, 58 Samgaksan-ro, Gangbuk-gu, Seoul 01022, Republic of Korea.

e-mail: wksong@nrc.go.kr,wonksong@gmail.com 
upper limbs of a group of nine chronic stroke patients. In addition, Staubli et al. (2009) investigated the effect of intensive arm training with rehabilitation robots and found that three of four stroke patients showed significant improvements on motor performance. However, although robotic devices have been used for upper extremity rehabilitation after stroke (Mehrholz et al. 2008), scientific evidence for their benefits is still insufficient. In particular, despite some studies reporting the effectiveness of robotic devices in improving upper limb motor function and strength after stroke (Lo et al. 2010; Basteris et al. 2014), it is unclear whether the improvements in function and strength transfer to activities of daily living.

Therefore, the purpose of the current study was to assess whether a robot-assisted reach training program using a whole arm manipulator (WAM) could improve upper extremity kinematic performance and functional movement of chronic stroke patients. We hypothesized that chronic stroke patients would show improvements in upper extremity kinematic performance and functional movement after four weeks of robot-assisted reach training using WAM. The overall goal of this study was to provide a reference point for a subsequent randomized controlled trial.

\section{Subjects and Methods}

Subjects

Twelve chronic stroke patients participated in this study. Subjects were screened according to the following inclusion and exclusion criteria. Inclusion criteria were 1) hemiparesis from a single stroke occurring at least six months prior, 2) sufficient cognition to follow simple instructions and understand the purpose of the study (Mini-Mental State Examination [MMSE] score $>18$ points) (Masiero et al. 2011), 3) absence of a musculoskeletal condition that could affect the ability to sit safely, and 4) absence of hemispatial neglect. Exclusion criteria were 1) participation in other studies or rehabilitation programs, 2) shoulder subluxation or pain in the upper limbs, or 3) spasticity (Modified Ashworth Scale score $>2$ ) (Frisoli et al. 2012). Two of the 12 potential subjects were excluded because they failed to meet the inclusion criteria. Ultimately, 10 subjects (6 male, 61.5 years; MMSE: 27.0; onset duration: 8.9 years) were included in the study. Clinical and demographic data with baseline clinical assessment at enrollment are presented in Table 1. Subjects were recruited from the local community. They were not presently receiving any rehabilitation services such as, physical or occupational therapy. For clinical data at baseline, the Modified Ashworth Scale (MAS) is considered the primary clinical measure of muscle spasticity for patients with neurological conditions. In addition, the Medical Research Council (MRC) scale is considered the primary clinical measure of muscle strength for patients with neurological conditions. In this study, we used only the shoulder portion of the MRC scale to evaluate the participations.

\section{Procedure}

A single group-case design was used to investigate whether robot-assisted reach training could improve upper extremity kinematic performance and functional movement. The 10 subjects were briefed on the experimental procedure, and written consent was collected from all subjects prior to the experiment. Human subject ethi- cal approval was obtained from the relevant committee of the Korea National Rehabilitation Center Institutional Review Board (NRC2012-05-035) prior to conducting the experiment.

All subjects underwent the training program two times a week for four weeks. A single training session was 40 minutes in duration and was led by the same assistants. All 10 subjects completed the training sessions.

Subjects were assessed for functional movement with the Action Research Arm Test. In addition, upper extremity kinematic performance was measured using movement velocity for upper limb reaching. Measurements of functional movement and extremity kinematic performance were performed three times: at baseline, during intervention (at 2 weeks), and post-intervention.

\section{Study protocol (robot-assisted reach training program)}

The robot-assisted reach training program was conducted in a test bed. The training test bed consisted of one whole arm manipulator (WAM) (Barrett Technology, Inc., Newton, MA, USA) and one projective display device (Fig. 1A). The WAM was set at seven degrees of freedom for the shoulder, elbow, and wrist joints during the training sessions. The WAM provides a highly back-drivable motion that helps the user reach the desired sphere using point-topoint movements. In addition, the WAM can assist when either proving weight support or proving force is the aim of task completion. A 120 -inch projective display attached to the front of the test bed was

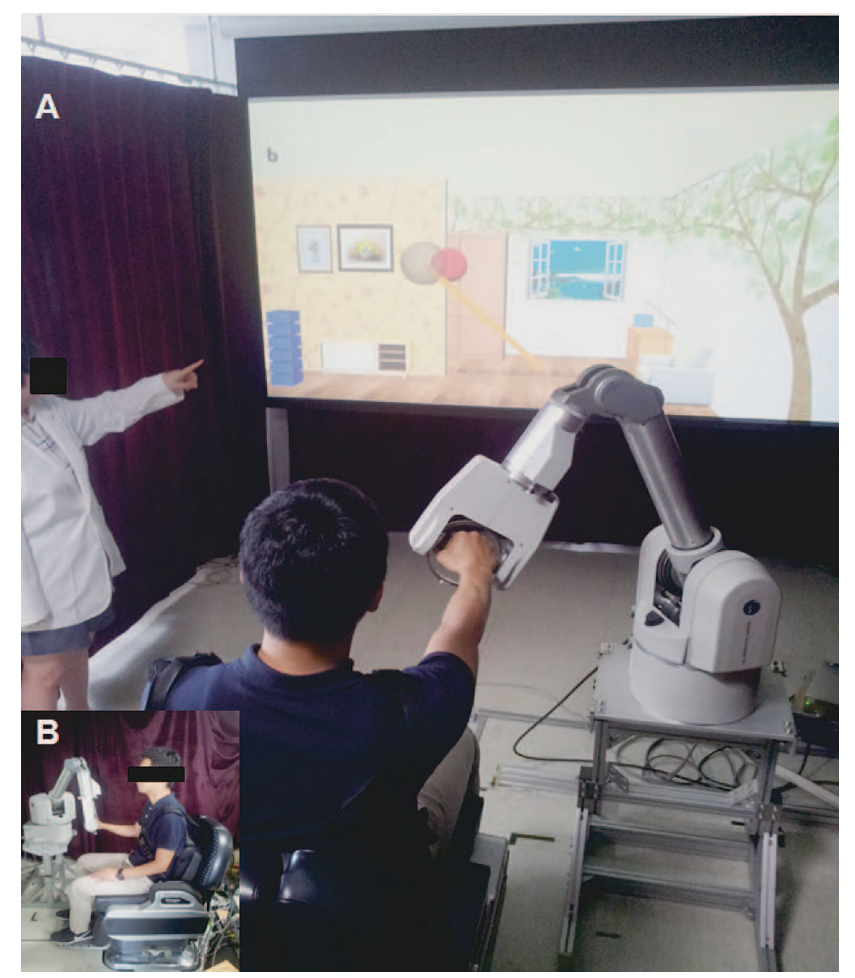

Fig. 1. Test bed for the robot-assisted reach training program (A) and start position of the robot-assisted reach training (B).

The training test bed is consisted of whole arm manipulator (WAM) and a projective display. The WAM was set at seven degrees of freedom for the shoulder, elbow, and wrist joints during the training sessions. The WAM provides a highly back-drivable motion that helps the user reach the desired sphere using point-to-point movements. 


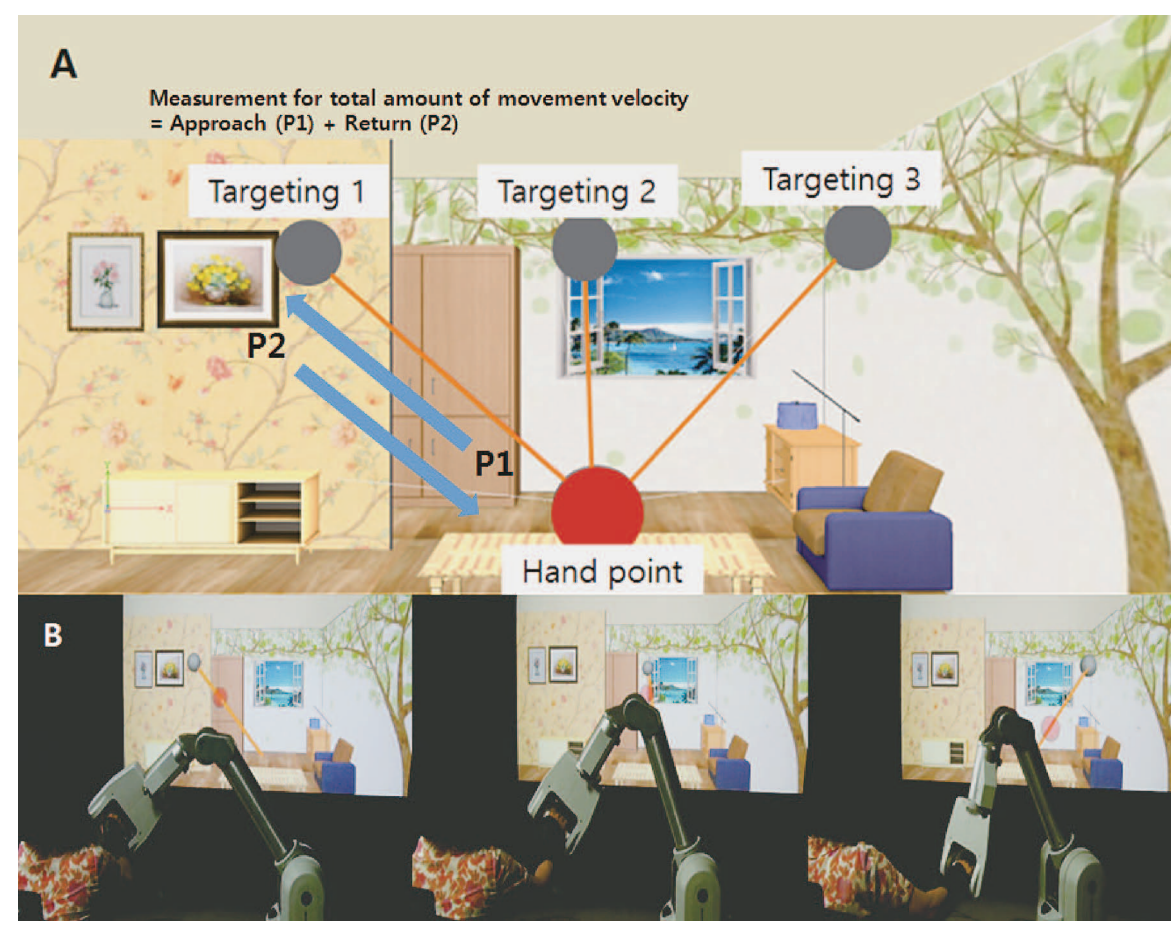

Fig. 2. Robot-assisted reach training protocol (the screenshot of the projective display (A) and the robot-assisted reach training in the test bed (B)).

Upper extremity kinematic analysis was based on the performance of a reaching movement toward targets. Performance was analyzed in accordance with movement velocity. Movement velocity was recorded while performed reaching from the hand point to the target point (Approach: P1) and return back to the hand point (Return: P2). Red and gray balls appeared on the projective display and subject performed reaching movement towards to a target in three directions (A). The red ball is linked with hand movements of a subject. The subjects' movements were executed to reach different targets placed at ipsilateral, central and contralateral positions (B).

used to provide suitable visual and auditory feedback to the user.

Subjects performed the robot-assisted reach training exercises while wearing the WAM and sitting on a chair. In addition, subjects wore a trunk-fixed belt to minimize compensatory movement and the likelihood of emergency situations (Fig. 1B). Once the training began, red and gray balls appeared on the projective display and the subjects performed reaching movements toward targets in threedimensional space in three directions (Fig. 2). The red ball was linked with upper limb movements of the subject, and auditory feedback was provided when the red and gray balls matched. One task consisted of three phases: moving toward the target, manipulating the target, and returning from the target. While performing the training exercises, the velocity from the starting point to the target point and the target point to the starting point was recorded.

To prevent accidents due to fatigue, an assistant stood nearby and emergency stop devices were installed. All subjects participated in the robot-assisted reach training program for 40 minutes per day, 2 times a week, for 4 weeks. Rest breaks were allowed if requested, but were not included in the overall walking time.

\section{Outcome measurements}

Upper extremity kinematic performance: Upper extremity kinematic analysis was based on the performance of a reaching movement toward targets. Performance was analyzed in accordance with movement velocity. The formula used to determine movement velocity was as follows:

$$
\text { movement velocity }(\mathrm{mm} / \mathrm{sec})=\frac{\text { movement distance }(\mathrm{mm})}{\text { movement time }(\mathrm{sec})}
$$

Movement time was defined as the time from the starting point to the target point and the time from the target point to the starting point. Movement distance was defined as the distance from the starting point to the target point and the distance from the target point to the starting point. Movement velocity was recorded while the participation reached from the hand point to the target point and returned to the hand point. The subjects' movements were executed to reach different targets placed in ipsilateral, central, and contralateral positions. Data were transferred to MATLAB software for further analysis.

Functional movement: The Action Research Arm Test (ARAT) was used to measure functional movement. ARAT is designed to assess upper limb disability through the assessment of four basic movements, including primary grasp, grip, pinch, and gross movements of flexion and extension at the elbow and shoulder. Each test is graded on a four-point scale, from 0 (unable to complete any part of the hand or arm movement) to 3 (normal performance), yielding a maximum score of 57 (Yozbatiran et al. 2008).

\section{Data analysis}

Data analysis was performed using SPSS, Version 21.0 (SPSS Inc., Chicago, IL, USA). The Shapiro-Wilk test was used to confirm the normal distribution of all outcome variables. All variables (functional movement and upper extremity kinematic performance in three directions) were normally distributed; therefore, we chose one-way 
analysis of variance with repeated measures using a within-subject factor at three levels (pre-training and 2 and 4 weeks after training). If a significant main effect was found, paired $t$-test was used to determine between which comparisons the differences existed. All outcomes are expressed as mean values and standard deviations. Statistical significance was accepted for $\mathrm{P}<0.05$.

\section{Results}

A summary of the general characteristics of the 10 subjects who fulfilled the inclusion criteria is shown in
Table 1. Table 2 and Fig. 3 show the changes in upper extremity kinematic performance (movement velocity) and functional movement (ARAT), respectively. Regarding movement velocity for target 1 , after four weeks of training, significant improvement in upper extremity movement velocity was observed $(\mathrm{P}<0.05)$. Regarding movement velocity for target 2 , after two and four weeks of training, significant improvement in upper extremity movement velocity was observed $(\mathrm{P}<0.05)$. Regarding movement velocity for target 3 , after two and four weeks of training,

Table 1. Subjects characteristics of the study $(\mathrm{N}=10)$.

\begin{tabular}{|c|c|c|c|c|c|c|c|c|c|c|}
\hline Subjects & $\begin{array}{c}\text { Sex } \\
(\mathrm{M} / \mathrm{F})\end{array}$ & $\begin{array}{c}\text { Age } \\
\text { (years) }\end{array}$ & $\begin{array}{c}\text { Time since } \\
\text { stroke } \\
\text { (years) }\end{array}$ & $\begin{array}{l}\text { Height } \\
(\mathrm{cm})\end{array}$ & $\begin{array}{l}\text { Weight } \\
(\mathrm{kg})\end{array}$ & $\begin{array}{c}\text { Etiology } \\
(\mathrm{I} / \mathrm{H})\end{array}$ & $\begin{array}{l}\text { Paretic side } \\
\quad(\mathrm{L} / \mathrm{R})\end{array}$ & $\begin{array}{c}\text { MAS } \\
(0 / 1 / 1+)\end{array}$ & $\begin{array}{c}\text { MRC } \\
\text { (shoulder) } \\
(3 / 4 / 5)\end{array}$ & $\begin{array}{l}\text { MMSE } \\
\text { (scores) }\end{array}$ \\
\hline 1 & M & 75 & 13 & 164.8 & 64.5 & I & L & 0 & 4 & 29 \\
\hline 2 & M & 61 & 10 & 184.2 & 87.7 & $\mathrm{H}$ & $\mathrm{L}$ & 1 & 4 & 27 \\
\hline 3 & M & 58 & 9 & 166.0 & 71.0 & I & L & $1+$ & 5 & 27 \\
\hline 4 & $\mathrm{~F}$ & 59 & 6 & 156.5 & 52.0 & I & $\mathrm{R}$ & 0 & 3 & 29 \\
\hline 5 & $\mathrm{~F}$ & 68 & 10 & 158.2 & 67.3 & I & $\mathrm{R}$ & $1+$ & 3 & 24 \\
\hline 6 & M & 58 & 8 & 171.5 & 65.5 & $\mathrm{H}$ & L & 1 & 4 & 24 \\
\hline 7 & M & 61 & 9 & 163.0 & 59.8 & $\mathrm{H}$ & $\mathrm{R}$ & 1 & 3 & 27 \\
\hline 8 & M & 62 & 5 & 165.6 & 55.6 & $\mathrm{H}$ & $\mathrm{R}$ & 0 & 4 & 29 \\
\hline 9 & $\mathrm{~F}$ & 62 & 11 & 150.3 & 46.4 & $\mathrm{H}$ & L & 1 & 3 & 24 \\
\hline 10 & $\mathrm{~F}$ & 51 & 8 & 141.9 & 47.0 & $\mathrm{H}$ & $\mathrm{R}$ & 1 & 3 & 30 \\
\hline $\begin{array}{l}\text { M (SD) or } \\
\text { numbers }\end{array}$ & $6 / 4$ & $61.50(6.38)$ & $8.90(2.33)$ & $162.20(11.57)$ & $61.68(12.47)$ & $4 / 6$ & $5 / 5$ & $(3 / 5 / 2)$ & $(5 / 4 / 1)$ & $27.00(2.30)$ \\
\hline
\end{tabular}

Values are expressed as mean (standard deviation) or numbers.

The MAS is considered the primary clinical measure of muscle spasticity in patients with neurological conditions. MRC scale is considered the clinical measure of muscle strength in patients with neurological conditions. This study used only the shoulder part as characteristics of the subjects.

A summary of the general characteristics of the 10 subjects with chronic stroke who fulfilled the inclusion criteria for the study is shown in Table 1.

H, hemorrhage; I, infarction; L, left; R, right; MAS, Modified Ashworth Scale; MRC (shoulder), Medical Research Council scale (shoulder); MMSE, Mini-Mental State Examination.

Table 2. Changes of the upper extremity kinematic performance and functional movement $(\mathrm{N}=10)$.

\begin{tabular}{|c|c|c|c|c|c|c|c|}
\hline \multicolumn{2}{|c|}{ Variables } & Baseline & $\begin{array}{l}\text { After training } \\
\quad(2 \text { week })\end{array}$ & $\mathrm{D}^{1}$ & $\begin{array}{l}\text { After training } \\
\quad(4 \text { week })\end{array}$ & $\mathrm{D}^{2}$ & $\mathrm{~F}(\mathrm{P})$ for time factor \\
\hline \multicolumn{8}{|c|}{ Kinematic performance } \\
\hline \multirow{3}{*}{$\begin{array}{c}\text { movement } \\
\text { velocity } \\
(\mathrm{mm} / \mathrm{sec})\end{array}$} & target 1 & $5.36(2.34)$ & $7.79(4.88)$ & $2.43(1.03)^{*}$ & $7.03(3.59)$ & $1.67(0.51)^{\#}$ & $5.011(0.039)$ \\
\hline & target 2 & $4.17(2.06)$ & $5.49(2.79)$ & $1.32(0.28)^{*}$ & $5.30(2.70)$ & $1.13(0.27)^{\#}$ & $14.588(<0.000)$ \\
\hline & target 3 & $3.48(1.98)$ & $4.49(2.49)$ & $1.00(0.22)^{*}$ & $4.38(2.52)$ & $0.89(0.20)^{\#}$ & $13.698(<0.000)$ \\
\hline \multicolumn{8}{|c|}{ Functional movement } \\
\hline \multicolumn{2}{|c|}{ ARAT (scores) } & $25.60(12.22)$ & $31.90(12.52)$ & $6.30(2.00)^{*}$ & $35.80(12.68)$ & $10.20(3.08)^{\#}$ & $4.867(0.041)$ \\
\hline
\end{tabular}

Values are expressed as mean (standard deviation).

${ }^{1}$ Difference between baseline and after training ( 2 week).

${ }^{2}$ Difference between baseline and after training (4 week).

*Significant differences between baseline and 2 weeks after training, $\mathrm{P}<0.05$.

"Significant differences between baseline and 4 weeks after training, $\mathrm{P}<0.05$.

Table 2 shows the changes of the upper extremity kinematic performance and functional movement. After 4 weeks of robot assisted reaching training for 3 directions, significant improvement on upper extremity kinematic performance and functional movement were observed in individuals with chronic stroke $(\mathrm{P}<0.05)$.

ARAT, Action Research Arm Test. 


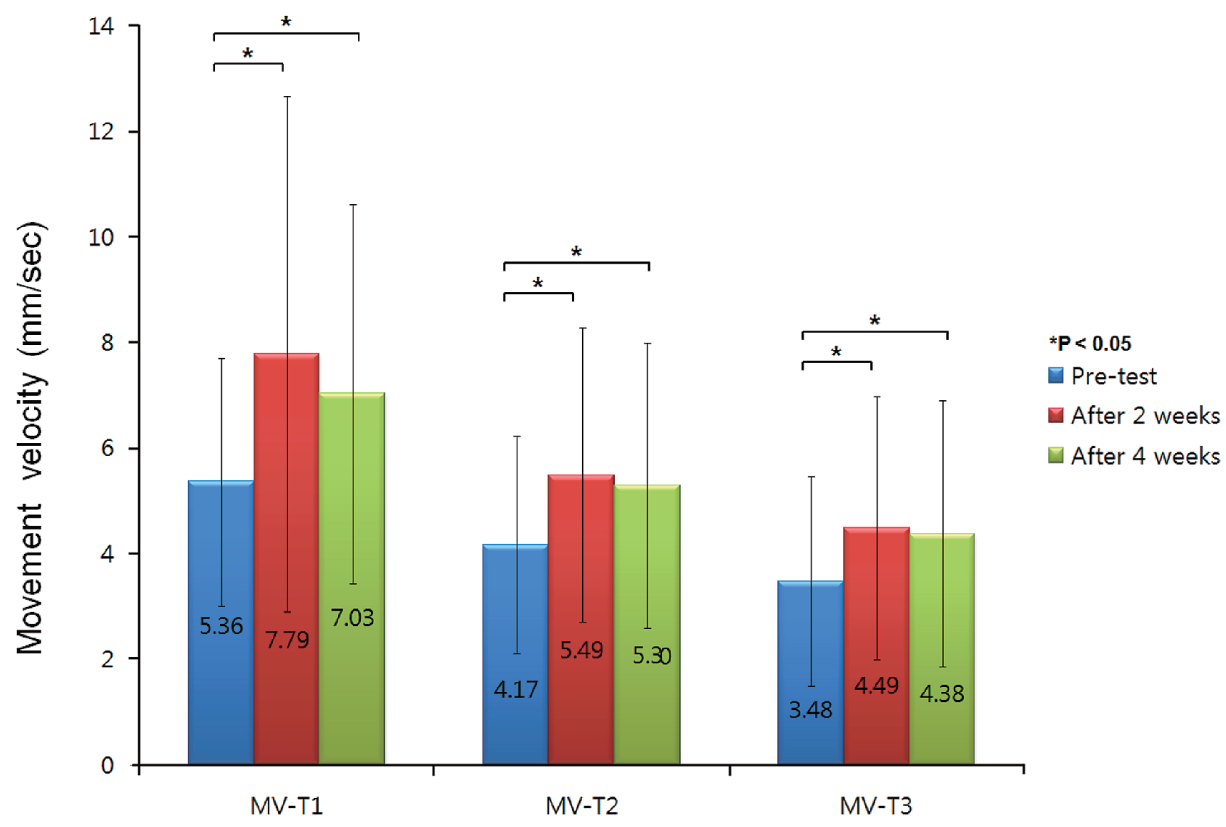

Fig. 3. Changes of movement velocity during robot-assisted reach training.

After 4 weeks of robot-assisted reach training for 3 directions, significant improvement on upper extremity kinematic performance (movement velocity) was observed in individuals with chronic stroke $(\mathrm{P}<0.05)$. Interestingly, upper extremity kinematic performance (movement velocity) for 3 directions showed highest improvement after 2 weeks of intervention.

MV-T1, movement velocity for target 1; MV-T2, movement velocity for target 2; MV-T3, movement velocity for target 3.

significant improvement in upper extremity movement velocity was observed $(\mathrm{P}<0.05)$. In addition, after two and four weeks of training, subjects showed significant improvement in upper extremity movement velocity on the ARAT $(\mathrm{P}<0.05)$.

\section{Discussion}

Stroke is a risk factor associated with chronic impairment of upper limb function (Nakayama et al. 1994; Mehrholz et al. 2008). Therefore, restoration of upper limb function is an essential goal of stroke rehabilitation. According to a published survey on robotic devices for upper limb rehabilitation, the objective of robotic devices for upper limb rehabilitation is starting to move away from validating device safety to refining therapeutic benefit (Maciejasz et al. 2014). Therefore, the current study was conducted in order to investigate the efficacy of a robotassisted reach training program on upper extremity kinematic performance and functional movement in chronic stroke patients. After four weeks of robot-assisted training, improvements in upper extremity kinematic performance and functional movement were observed in 10 individuals recovering from stroke.

In a hospital setting, various clinical assessment tools are used to determine the effectiveness of upper limb rehabilitation using a robotic device (Mehrholz et al. 2012). However, upper extremity dysfunction is often more severe than lower limb dysfunction (Pandian and Arya 2014). In addition, clinical assessment alone is insufficient to accu- rately evaluate the degree of impairment (Kim et al. 2014). Recent studies of stroke rehabilitation using robotic devices have focused on kinematic movement measurement (Bosecker et al. 2010; Kim et al. 2014; Rohafza et al. 2014). Kinematic movement measurement provides information regarding the ability to move the extremities through space (Rohafza et al. 2014) and kinematic movement measurement is considered suitable to obtain specific and accurate motion data (Kim et al. 2014). In particular, according to recent studies of robot-assisted training for stroke patients, the combination of kinematic assessment and traditional clinical scales may allow for quantitative evaluation of motor impairment (Patterson et al. 2011; van Dokkum et al. 2014). Thus, this study evaluated upper limb kinematic movement when performing a reaching task in tandem with the use of clinical scales.

The movement pattern of the upper limb is facilitated by complex structure. In particular, stroke patients have an abnormal movement pattern of the paretic upper limb due to motor dysfunction, paresthesia, and spasticity (Morris et al. 1997). Thus, many previous studies of upper limb training have emphasized that stroke rehabilitation training paradigm is needed to provide more detailed information about the components of movement (Basteris et al. 2014; Pollock et al. 2014). In particular, it was highlighted that specific and repetitive tasks are most effective in acceleration of neuroplasticity of the brain (Hubbard et al. 2009; Timmermans et al. 2010). Liao et al. (2012) reported that repetitive robot-assisted upper limb rehabilitation can 
improve motor function and arm activity of stroke patients, and Frisoli et al. (2012) demonstrated that robotic-assisted rehabilitation training including reaching and spatial antigravity movements induced decrement of movement time and improvement of motor recovery and functional movement. Findings from the current study are consistent with previous studies; we believe that the specific and repetitive tasks implemented during robot-assisted reach training led to an improvement of functional movement of the upper extremity in stroke patients.

Interestingly, another notable result was observed in this study: functional movement (ARAT) constantly improved during the four-week intervention. On the other hand, kinematic performance (movement velocity) in three directions showed the highest degree of improvement after two-week intervention (Fig. 3). This result indicates that the two-week robot-assisted reach training may be sufficient for achieving the beneficial effects. However, functional movement patterns require fast movement to solve problems as well as the ability to perform accurately (Hubbard et al. 2009). We think that participants in our study focused on the accuracy of their movements rather than movement velocity after two weeks of intervention. This was confirmed by the continuous improvement of functional movement during the intervention period.

The protocol associated with robot-assisted reach training is another important issue suggested by the current study. Functional movement of the upper extremity after stroke is one of the most important factors for performing the independent activities of daily living (Mercier et al. 2001). Because the reaching motion of the upper limb in various directions enables independent activity (Kim et al. 2014), many previous studies of robot therapy have developed training protocols related to goal-directed reaching movements (Burgar et al. 2011; Frisoli et al. 2012). In addition, training tasks related to the activities of daily living can provide substantial therapeutic effects and induce strong motivation to recover from injury (Hubbard et al. 2009). Thus, the current study conducted point-to-point reaching movements to provide specific and repetitive tasks similar to the actions associated with the activities of daily living (eating, drinking, etc.). However, since motion of the upper extremity cannot be represented by just a single activity, we think that further studies related to various daily living activities must be conducted.

In conclusion, the findings of the current study demonstrated the positive effects of short-term robot-assisted reach training on upper limb kinematic performance as well as functional movement in individuals with chronic stroke. However, this study has some limitations. First, despite the fact that improvement of upper extremity function was observed in all patients compared to baseline, this study had no separate control group. In addition, this study included only high-functioning stroke patients. Thus, the results of the current study cannot be generalized to all stroke patients. However, since the purpose of the current study was to assess whether a robot-assisted reach training program could improve upper extremity functional movement of chronic stroke patients, the findings of this study may provide basic information for a subsequent randomized controlled trial. In addition, since data for the clinical evaluation of robotic devices for upper limb rehabilitation are still sparse, a randomized controlled trial using a larger sample size will be necessary. Second, it is known that the inertia level of a robotic device may affect the difficulty level associated with certain movements. The higher inertia of the WAM $(25-27 \mathrm{~kg})$ in this study may have interfered with the training regimen. Thus, further studies of robotassisted reach training applied with low inertia will also be required.

\section{Acknowledgments}

This study was supported by the Research Program (NRCRI13-A-05, NRCTR-IN13004, NRCTR-IN14006, NRCTR-IN15005) of the Korea National Rehabilitation Center, Ministry of Health \& Welfare, Republic of Korea. We thank Mr. Ji Young Jeong, Ms. Yale Kim, Dr. Jung Yoon Kim, Dr. Joon-Ho Shin, Dr. Dae-Sung Park, Mr. Jun-Yong Song, Mr. Soon-Joon Jeong, Ms. Hwi-Young Lee, Mr. Byung-Woo Ko, Dr. Jongbae Kim, Dr. Dong-Ah Kim, and Dr. Hyun Choi for their assistance and comments during the development of the manuscript.

\section{Conflict of Interest}

The authors declare no conflict of interest.

\section{References}

Basteris, A., Nijenhuis, S.M., Stienen, A.H., Buurke, J.H., Prange, G.B. \& Amirabdollahian, F. (2014) Training modalities in robot-mediated upper limb rehabilitation in stroke: a framework for classification based on a systematic review. $J$. Neuroeng. Rehabil., 11, 111.

Bosecker, C., Dipietro, L., Volpe, B. \& Krebs, H.I. (2010) Kinematic robot-based evaluation scales and clinical counterparts to measure upper limb motor performance in patients with chronic stroke. Neurorehabil. Neural Repair, 24, 62-69.

Burgar, C.G., Lum, P.S., Scremin, A.M., Garber, S.L., Van der Loos, H.F., Kenney, D. \& Shor, P. (2011) Robot-assisted upper-limb therapy in acute rehabilitation setting following stroke: Department of Veterans Affairs multisite clinical trial. J. Rehabil. Res. Dev., 48, 445-458.

Frisoli, A., Procopio, C., Chisari, C., Creatini, I., Bonfiglio, L., Bergamasco, M., Rossi, B. \& Carboncini, M.C. (2012) Positive effects of robotic exoskeleton training of upper limb reaching movements after stroke. J. Neuroeng. Rehabil., 9, 36.

Hubbard, I.J., Parsons, M.W., Neilson, C. \& Carey, L.M. (2009) Task-specific training: evidence for and translation to clinical practice. Occup. Ther. Int., 16, 175-189.

Kim, K., Song, W.K., Lee, J., Lee, H.Y., Park, D.S., Ko, B.W. \& Kim, J. (2014) Kinematic analysis of upper extremity movement during drinking in hemiplegic subjects. Clin. Biomech., 29, 248-256.

Kolominsky-Rabas, P.L., Weber, M., Gefeller, O., Neundoerfer, B. \& Heuschmann, P.U. (2001) Epidemiology of ischemic stroke subtypes according to TOAST criteria: incidence, recurrence, and long-term survival in ischemic stroke subtypes: a population-based study. Stroke, 32, 2735-2740.

Kwakkel, G., Kollen, B.J., van der Grond, J. \& Prevo, A.J. (2003) Probability of regaining dexterity in the flaccid upper limb: 
impact of severity of paresis and time since onset in acute stroke. Stroke, 34, 2181-2186.

Liao, W.W., Wu, C.Y., Hsieh, Y.W., Lin, K.C. \& Chang, W.Y. (2012) Effects of robot-assisted upper limb rehabilitation on daily function and real-world arm activity in patients with chronic stroke: a randomized controlled trial. Clin. Rehabil., 26, 111-120.

Lo, A.C., Guarino, P.D., Richards, L.G., Haselkorn, J.K., Wittenberg, G.F., Federman, D.G., Ringer, R.J., Wagner, T.H., Krebs, H.I., Volpe, B.T., Bever, C.T., Bravata, D.M., Duncan, P.W., Corn, B.H., Maffucci, A.D., et al. (2010) Robot-assisted therapy for long-term upper-limb impairment after stroke. N. Engl. J. Med., 362, 1772-1783.

Maciejasz, P., Eschweiler, J., Gerlach-Hahn, K., Jansen-Troy, A. \& Leonhardt, S. (2014) A survey on robotic devices for upper limb rehabilitation. J. Neuroeng. Rehabil., 11, 3.

Masiero, S., Armani, M. \& Rosati, G. (2011) Upper-limb robotassisted therapy in rehabilitation of acute stroke patients: focused review and results of new randomized controlled trial. J. Rehabil. Res. Dev., 48, 355-366.

Mehrholz, J., Hadrich, A., Platz, T., Kugler, J. \& Pohl, M. (2012) Electromechanical and robot-assisted arm training for improving generic activities of daily living, arm function, and arm muscle strength after stroke. Cochrane Database Syst. Rev., 13, CD006876.

Mehrholz, J., Platz, T., Kugler, J. \& Pohl, M. (2008) Electromechanical and robot-assisted arm training for improving arm function and activities of daily living after stroke. Cochrane Database Syst. Rev., 8, CD006876.

Mercier, L., Audet, T., Hebert, R., Rochette, A. \& Dubois, M.F. (2001) Impact of motor, cognitive, and perceptual disorders on ability to perform activities of daily living after stroke. Stroke, 32, 2602-2608.

Morris, D.M., Crago, J.E., Deluca, S.C., Pidikiti, R.D. \& Taub, E. (1997) Constraint-induced movement therapy for moter recovery after stroke. NeuroRehabilitation, 9, 29-43.

Nakayama, H., Jorgensen, H.S., Raaschou, H.O. \& Olsen, T.S. (1994) Recovery of upper extremity function in stroke patients: the Copenhagen Stroke Study. Arch. Phys. Med.
Rehabil., 75, 394-398.

Pandian, S. \& Arya, K.N. (2014) Stroke-related motor outcome measures: do they quantify the neurophysiological aspects of upper extremity recovery? J. Bodyw. Mov. Ther., 18, 412-423.

Patterson, T.S., Bishop, M.D., McGuirk, T.E., Sethi, A. \& Richards, L.G. (2011) Reliability of upper extremity kinematics while performing different tasks in individuals with stroke. J. Mot. Behav., 43, 121-130.

Peter, O., Fazekas, G., Zsiga, K. \& Denes, Z. (2011) Robot-mediated upper limb physiotherapy: review and recommendations for future clinical trials. Int. J. Rehabil. Res., 34, 196-202.

Pollock, A., Farmer, S.E., Brady, M.C., Langhorne, P., Mead, G.E., Mehrholz, J. \& van Wijck, F. (2014) Interventions for improving upper limb function after stroke. Cochrane Database Syst. Rev., 12, CD010820.

Rohafza, M., Fluet, G.G., Qiu, Q. \& Adamovich, S. (2014) Correlation of reaching and grasping kinematics and clinical measures of upper extremity function in persons with stroke related hemiplegia. Conf. Proc. IEEE Eng. Med. Biol. Soc., 2014, 3610-3613.

Staubli, P., Nef, T., Klamroth-Marganska, V. \& Riener, R. (2009) Effects of intensive arm training with the rehabilitation robot ARMin II in chronic stroke patients: four single-cases. $J$. Neuroeng. Rehabil., 17, 46.

Timmermans, A.A., Spooren, A.I., Kingma, H. \& Seelen, H.A. (2010) Influence of task-oriented training content on skilled arm-hand performance in stroke: a systematic review. Neurorehabil. Neural Repair, 24, 858-870.

Turner, D.L., Ramos-Murguialday, A., Birbaumer, N., Hoffmann, U. \& Luft, A. (2013) Neurophysiology of robot-mediated training and therapy: a perspective for future use in clinical populations. Front. Neurol., 13, 184.

van Dokkum, L., Hauret, I., Mottet, D., Froger, J., Metrot, J. \& Laffont, I. (2014) The contribution of kinematics in the assessment of upper limb motor recovery early after stroke. Neurorehabil. Neural Repair, 28, 4-12.

Yozbatiran, N., Der-Yeghiaian, L. \& Cramer, S.C. (2008) A standardized approach to performing the action research arm test. Neurorehabil. Neural Repair, 22, 78-90. 